\title{
ANATOMÍA MACROSCÓPICA, IRRIGACIÓN Y DRENAJE VENOSO DEL APARATO REPRODUCTOR FEMENINO DE LA LLAMA (Lama glama)
}

\author{
Macroscopic Anatomy, Irrigation and Venous Drainage of Female \\ Reproductive Apparatus of Llama (LAMa glama)
}

Eric León M. ${ }^{1}$, Alberto Sato S., ${ }^{1,2}$ Miluska Navarrete Z. ${ }^{1}$, Jannet Cisneros S. ${ }^{1}$

\section{RESUMEN}

Se hizo la descripción anatómica del tracto reproductor femenino de la llama. Se usaron 4 llamas adultas. Macroscópicamente, la anatomía del aparato reproductor es morfológicamente similar a la del bovino, diferenciándose por la ausencia de ligamento intercornual y cotiledones, así como por la presentación de un tabique intercornual, como es el caso de la alpaca. La distribución de las arterias y venas que irrigaron y drenaron sangre a la cavidad pélvica y aparato reproductor presentaron en un primer tramo, a nivel de la arteria iliaca interna, una distribución vascular casi concordante con los patrones descritos en rumiantes; luego siguió un patrón similar al del equino. A nivel del aparato reproductor, los vasos sanguíneos adoptaron un patrón totalmente diferente al descrito en especies domésticas. Se encontraron arterias no antes descritas como la arteria vaginal caudal, la arteria vesical media, la arteria vaginal craneal, la arteria uterina dorsal con sus ramas lateral y medial, y la arteria arco cervical. A cada arteria encontrada le correspondió una vena satélite. El cuerno uterino izquierdo presentó una mejor irrigación dado que la arteria uterina derecha envió su rama medial derecha hacia el lado izquierdo del aparato reproductor; además, la arteria arco cervical estableció comunicación entre las arterias uterinas izquierda y derecha, pasando por la superficie ventral de la cérvix. Esta arteria puede emerger tanto de la misma arteria uterina como de su rama medial.

Palabras clave: anatomía, reproductor, irrigación, llama

\section{Abstract}

The anatomical description of the reproductive tract of the female llama was studied in four animals. Macroscopically, the reproductive system is morphologically similar to the cow. However, the difference is the absence of intercornual ligament and cotyledons, and the presence of an intercornual septum, as in the alpaca. The distribution of the arteries and veins that irrigated and drained the blood to and from the pelvic cavity and

\footnotetext{
${ }^{1}$ Laboratorio de Anatomía Animal y Fauna Silvestre, Facultad de Medicina Veterinaria, Universidad Nacional Mayor de San Marcos, Lima

${ }^{2}$ E-mail: albertosato2000@yahoo.com
} 
reproductive system presented a vascular distribution almost equal to the ruminant's pattern and then, they followed a pattern similar to that on the equine. At the reproductive system level, blood vessels adopted a totally different pattern from those described for domestic species. Some arteries had never been described such as the caudal vaginal artery, medium vesical artery, cranial vaginal artery, dorsal uterine artery with its lateral and medial branches, and the arch cervical artery. Each artery had the corresponding satellite vein. The left uterine horn presented a better irrigation as the right uterine artery send its medial right branch to the left side of the reproductive system; moreover, the arch cervical artery established communication between the left and right uterine arteries through the cervix ventral surface. This artery could emerge from the uterine artery itself as well as from its medial branch.

Key words: anatomy, reproductive, irrigation, llama

\section{INTRODUCCIÓN}

Los camélidos sudamericanos (CSA) representan un modelo de adaptación a las condiciones ambientales y nutricionales existentes en los Andes, lugares que constituyen su ambiente natural (Ayacucho Portal Regional Agrario, 2008). La llama es un animal de gran utilidad para el poblador altoandino, pues le proporciona abrigo a través de su fibra, carne y medio de transporte, así como un rendimiento de canal de 60\% (Sumar, 1981).

Se vienen realizando diversos estudios para mejorar la productividad de los CSA; sin embargo, se conoce muy poco acerca de las estructuras anatómicas que conforman el aparato reproductor femenino de la llama. Existen diversos mecanismos de control fisiológico, dependiendo de la especie, basados en la relación entre el sistema vascular y las estructuras que conforman el aparato reproductor; y para comprender estos mecanismos es fundamental reconocer la anatomía macroscópica, incluyendo la irrigación y drenaje venoso del aparato reproductor.

El caso de las CSA es muy especial, pues se reconoce que tienen una baja tasa de natalidad, principalmente debido a problemas de muerte embrionaria, sin que hasta la fecha se conozca las razones específicas. Por otro lado, todavía no tiene una comprensión cabal de la fisiología reproductiva de la llama, por ser un animal anatómicamente diferente a las especies domésticas comúnmente utilizadas en producción, como el vacuno y el equino (Ministerio de Agricultura, 2008).

Existen descripciones generales acerca de la anatomía macroscópica del aparato reproductor femenino de la alpaca (Sato et al., 1986), y para el caso de la llama se dispone de una mención descriptiva en sentido caudo-craneal (Smith et al., 1994); asimismo, se tiene un cuadro de medidas de las diferentes estructuras macroscópicas que componen el aparato reproductor de la llama y la alpaca (Fowler, 1998). Se conoce que el 90\% de alpacas presentan el cuerno uterino izquierdo más irrigado, y que hay una cierta similitud con el canino y el camello sobre el patrón de origen de las arterias que irrigan al útero de la alpaca. Además, el patrón de irrigación del útero de la alpaca es semejante al del camello (Sato et al., 1988).

La irrigación del aparato reproductor femenino en bovinos y rumiantes menores se origina en las arterias umbilicales a través de su rama uterina (antes denominada arteria uterina media), la arteria vaginal (antes denominada arteria urogenital) y la arteria pudenda interna; todas ellas originadas en la arteria iliaca interna, última rama de la arteria aorta. En el caso de los equinos, las arterias glútea caudal con su rama glútea craneal (originando a la arteria del clítoris) y pudenda 
interna (la que emite la arteria vaginal) son las encargadas de la irrigación del aparato reproductor de la yegua (Sisson et al., 2000).

En las alpacas, la iliaca interna origina a la pudenda interna de la cual nace la arteria vaginal (urogenital) distribuyendo ramas hacia la vejiga, útero y vagina. La arteria uterina se divide en arterias uterinas medial y lateral, las que se anastomosan con sus respectivas homólogas procedentes de la rama uterina de la arteria ovárica (Sato y Montoya, 1990).

Trabajos realizados en ovinos acerca de la vascularización arterial del ovario durante el ciclo estral (Vilá et al., 2007), demuestran un patrón macroscópico de irrigación arterial del aparato reproductor femenino similar al reportado en la alpaca (Sato y Montoya, 1990).

El objetivo del presente estudio fue realizar una descripción macroscópica del aparato reproductor femenino de la llama, así como de su irrigación y drenaje venoso.

\section{Materiales y MÉTOdos}

El estudio se realizó en el Laboratorio de Anatomía Animal de la Facultad de Medicina Veterinaria de la Universidad Nacional Mayor de San Marcos, Lima, durante los meses de junio a setiembre de 2008. Se emplearon cuatro llamas adultas en lactación, de aproximadamente 4 años, provenientes de la zona de Huancavelica. Dos llamas se encontraban gestantes.

Los animales fueron tranquilizados con promazil ( $0.15 \mathrm{mg} / \mathrm{kg}$ vía i.m.) y anestesiados con pentobarbital sódico (Halatal $\left.{ }^{\circledR}\right)(10 \mathrm{mg} /$ $\mathrm{kg}$ ). Adicionalmente, se inyectó lidocaína al $2 \%$ i.m. en la zona de incisión en el cuello para la sangría. La muerte ocurrió por sangrado a través de un piquete en la arteria carótida común y vena yugular externa. Se canuló la arteria carótida común para el embalsamamiento con solución conservadora en base a formol al 12\%, ácido fénico 3\%, glicerina al $8 \%$ y agua csp 100\%. Para este proceso, se usó una bomba aspirante-impelente a una presión de 150 mm de Hg.

La disección se hizo desde el plano superficial, abordando la piel, músculos, huesos y vasos sanguíneos, hasta el plano profundo en la cavidad pélvica. La disección del aparato reproductor y sus respectivas arterias, venas y medios de fijación, se hizo mediante el corte de dichas estructuras y separación de tejido conectivo.

\section{Resultados y DiscusióN}

El ligamento ancho uterino fija al aparato reproductor a la cavidad pélvica, tomando diferentes denominaciones, según las estructuras con las que se relaciona: El mesovario suspende a los ovarios desde una posición cráneo lateral, ubicados en la región sublumbar; el mesosálpinx, adherido a las trompas uterinas, fija estas estructuras a la pared abdominal; la bolsa ovárica, formada por la unión del mesovario y mesosálpinx a nivel del borde mesovárico del ovario, estructura bastante desarrollada en la llama; el ligamento propio del ovario, que fija el ovario al cuerno uterino; el mesometrio, que asienta en el borde dorsal de los cuernos uterinos y en los bordes laterales del cuerpo del útero; y el ligamento redondo del útero, que viene a ser una proyección del ligamento ancho en su superficie ventro craneal, a nivel de la inserción del mesovario.

La anatomía macroscópica del aparato reproductor femenino de la llama no presentó variaciones en cuanto a su forma entre las dos hembras vacías ni entre las dos hembras gestantes. En las hembras vacías, la anatomía y disposición del órgano reproductor en la cavidad pélvica y abdominal fue similar a las descripciones que existe para la alpaca (Sato y Montoya, 1990) y para el bovino (Sisson et al., 2000). Las características más resaltantes fueron la disposición 
de los cuernos uterinos dirigidos hacia el vientre del animal y la presencia de bolsas ováricas incompletas y desarrolladas. Además, no se apreciaron cotiledones en los cuatro especímenes disecados ni ligamento intercornual; sin embargo, internamente se evidenció la presencia de un tabique intercornual denominado velo uterino (Fig. 1).

El cuerno uterino grávido en el bovino desplaza los órganos abdominales hacia craneal disminuyendo el volumen expandible del estómago del animal. En las llamas, el compartimiento proximal del estómago ocupa el lado izquierdo del abdomen y hacia su borde derecho se ubica el compartimiento distal (Alzola et al., 2004). En las llamas gestantes el útero izquierdo grávido se acomodó dirigiéndose hacia el lado derecho, sin alterar la posición normal de los compartimientos del estómago.

La distribución de las arterias que irrigan la cavidad pélvica y aparato reproductor siguió un patrón diferente a los descritos en la Nómina Anatómica Veterinaria (2005) para especies domésticas; adoptando en un primer tramo, secuencias arteriales de forma similar que la especie bovina; para luego adoptar, en un segundo tramo, un patrón similar a la especie equina. Además, la distribución de los vasos sanguíneos a nivel del útero, presentó un patrón similar a los descritos en ovejas (Vilá et al., 2007) y en alpacas (Sato y Montoya, 1990).

La arteria iliaca interna (Fig. 2) es el tronco común del cual surgen las arterias responsables de la irrigación de la cavidad pélvica. En su primer trayecto, emitió la arteria umbilical y glútea craneal; patrón arterial que concuerda con el del bovino, excepto por la arteria iliolumbar, que en los especímenes disecados nació después de la arteria glútea craneal. Luego se emitieron la arteria glútea caudal y pudenda interna, y de esta última, se originó la arteria vaginal y demás ramas encargadas de la irrigación del aparato reproductor. Esta última descripción es casi concordante con la que se reporta en la es- pecie equina; sin embargo, cabe resaltar que se hallaron arterias no mencionadas para especies domésticas según la Nómina Anatómica Veterinaria (2005), como la arteria vaginal caudal, la arteria vesical media, la arteria vaginal craneal, la arteria uterina dorsal con sus respectivas ramas lateral y medial, y la arteria arco cervical (Fig. 3).

Se encontró una arteria y una vena no descritas con anterioridad: arteria arco cervical y vena arco cervical (Fig. 4). Estos vasos se encargan de la irrigación y drenaje de la cérvix, respectivamente. La arteria arco cervical nació de la arteria uterina medial derecha e izquierda y comunica ambos tramos arteriales. La vena arco cervical se ubicó en la superficie ventral de la cérvix y se dirigió lateralmente hacia las venas uterinas mediales derecha e izquierda. No se encontraron descripciones similares en el trabajo realizado en alpacas por Sato y Montoya (1990).

Otro hallazgo resaltante fue la distribución y anastomosis de las arterias y venas uterinas en la superficie ventral del aparato reproductor. La comunicación existente entre las arterias uterinas derecha e izquierda denota mayor aporte sanguíneo hacia el cuerno uterino izquierdo (Fig. 5); asimismo, las venas uterinas siguen el mismo recorrido que las arterias. Es a partir de aquí que la anatomía de las arterias del aparato reproductor femenino de la llama difiere sustancialmente de las descripciones anatómicas, tanto en el caso de bovinos como de los equinos.

\section{Conclusiones}

- La anatomía macroscópica del aparato reproductor femenino de la llama presenta mayor similitud morfológica con la especie bovina y la alpaca.

- En las llamas preñadas el útero izquierdo grávido se acomoda hacia el lado derecho, dejando del lado izquierdo al tracto digestivo. 


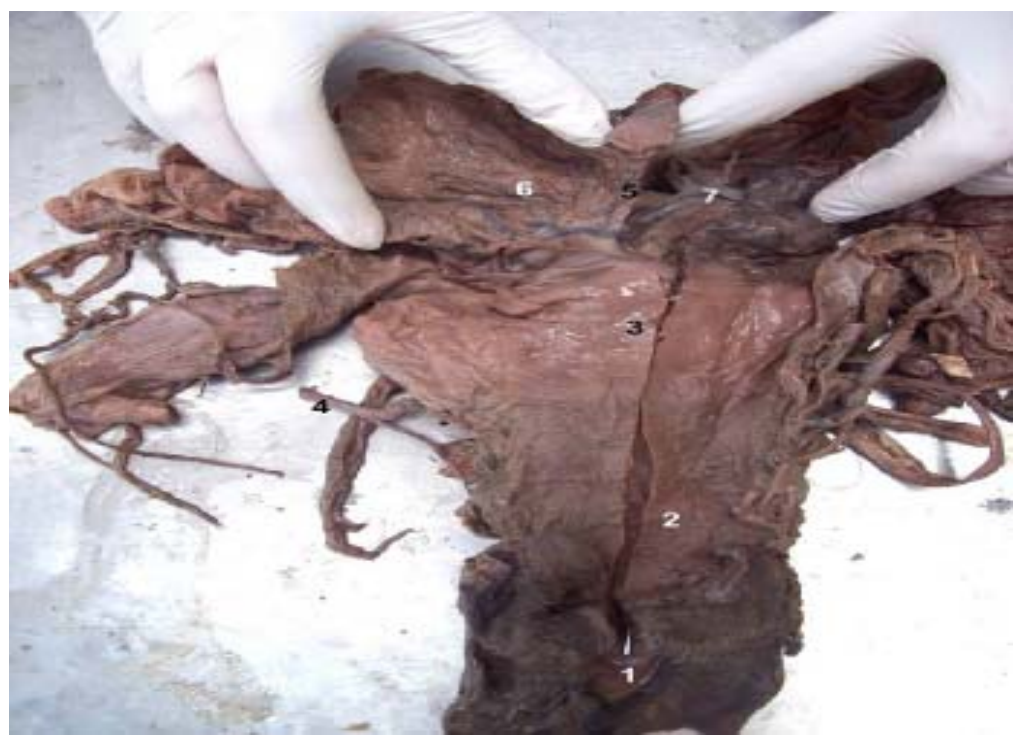

Figura 1. Vista dorso caudal del aparato reproductor femenino de la llama. 1. Vestíbulo vaginal; 2. Vagina; 3. Cérvix; 4. Arteria uterina derecha; 5. Velo uterino; 6. Cuerno uterino derecho; 7. Cuerno uterino izquierdo

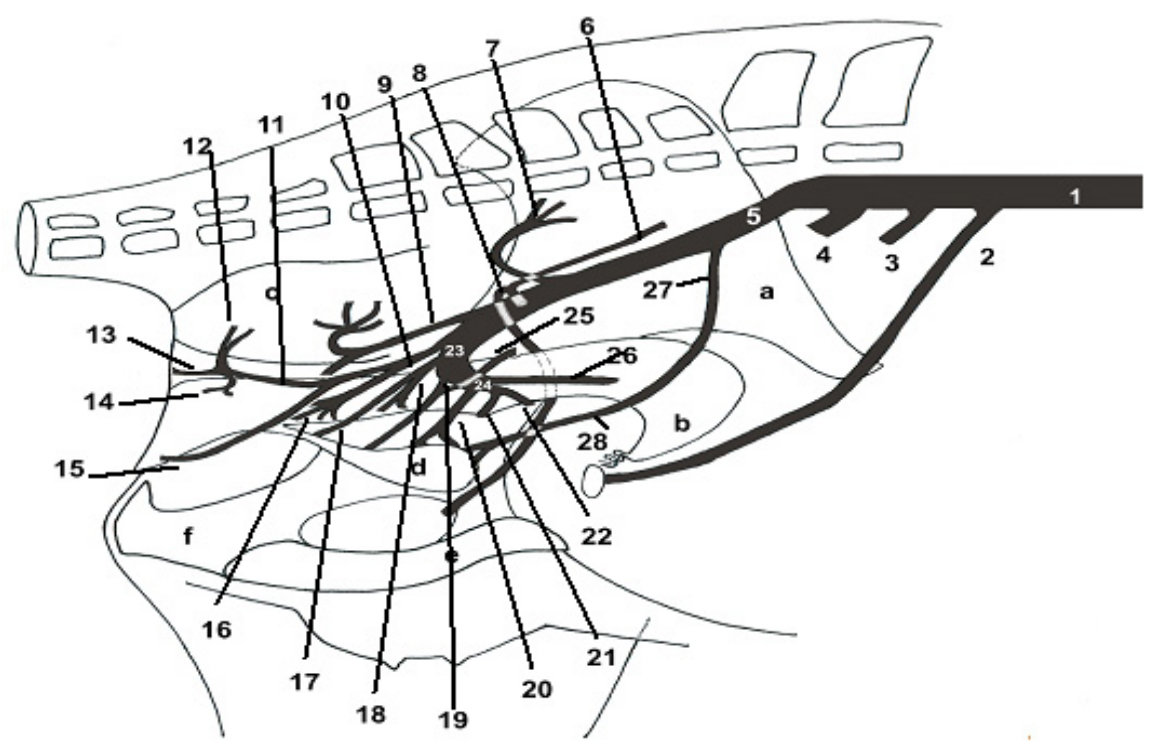

Figura 2. Representación gráfica de la irrigación arterial de la cavidad pélvica y aparato reproductor femenino de la llama en base a la descripción anatómica realizada. Vista medial. 1. Arteria Aorta abdominal; 2. Arteria Ovárica; 3. Arteria Mesentérica Caudal; 4. Arteria Iliaca Externa; 5. Arteria Iliaca Interna; 6. Arteria Iliolumbar; 7. Arteria Glútea Craneal: 8. Arteria Obturadora; 9. Arteria Glútea Caudal; 10. Arteria Pudenda Interna; 11. Arteria Perineal Ventral; 12. Arteria Rectal Caudal; 13. Arteria Dorsal Labial; 14. Arteria Vestibular; 15. Arteria Del Clítoris; 16. Arteria Vaginal Caudal; 17. Rama Uretral; 18. Arteria Vaginal Craneal; 19. Arteria Vesical Caudal; 20. Arteria Vesical Media; 21. Arteria Arco Cervical; 22. Arteria Uterina Medial Derecha; 23. Arteria Vaginal; 24. Arteria Uterina; 25. Arteria Uterina Dorsal; 26. Arteria Uterina Lateral Derecha; 27. Arteria Umbilical; 28. Arteria Vesical Craneal; a. Ilion; b. Cuerno Uterino; c. Ampolla Rectal; d. Vejiga; e. Pubis; f. Isquion. 


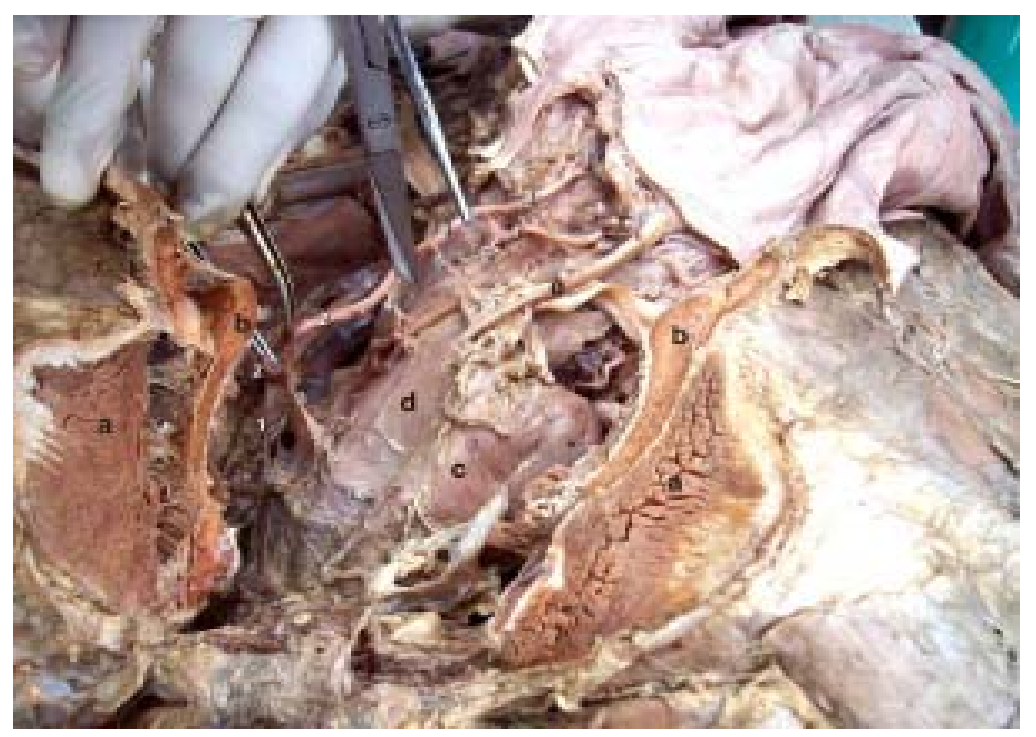

Figura 3. Vista ventro lateral de la cavidad pélvica mostrando las arterias que irrigan al aparato reproductor femenino de la llama. 1. Arteria Pudenda Interna; 2. Arteria Vaginal; 3. Arteria Vaginal Craneal; 4. Arteria Vesical Caudal; 5. Arteria Vesical Media; 6. Arteria Uterina Derecha; 7. Arteria Uterina Medial Derecha; 8. Arteria Uterina Lateral Derecha; 9. Arteria Arco Cervical; a. Músculo Gracilis; b. Sínfisis Isquio Púbica; c. Vejiga; d. Vagina; e. Uréter Derecho.

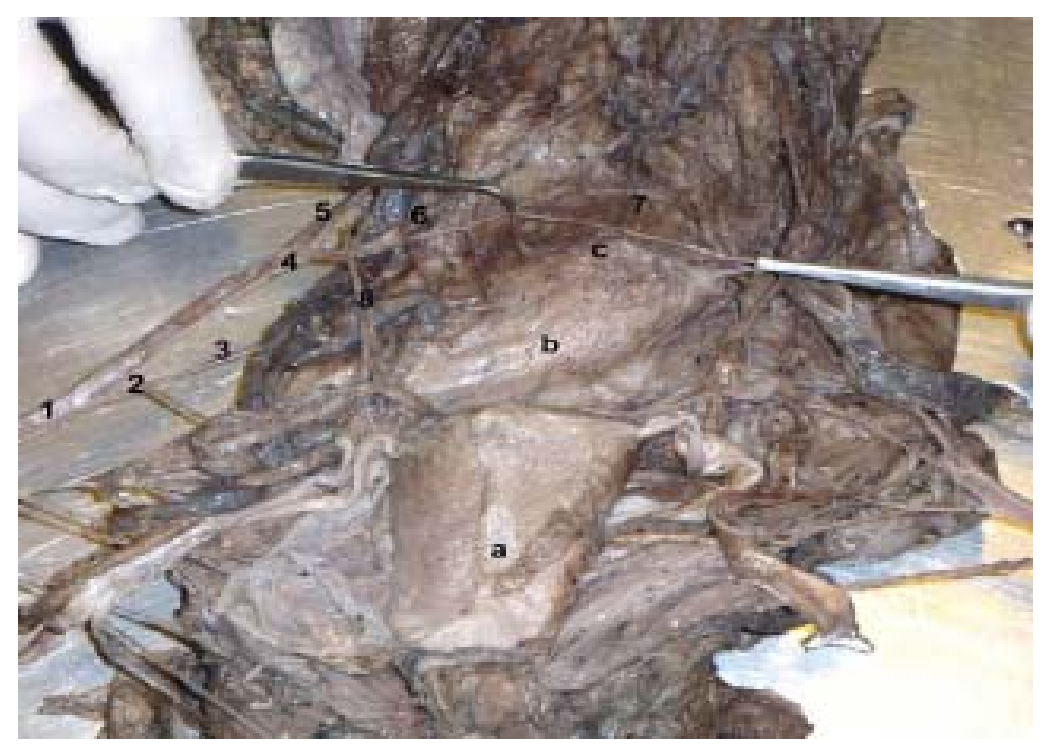

Figura 4. Vista ventro caudal del aparato reproductor femenino de la llama mostrando el patrón de irrigación arterial. 1. Arteria Vaginal Derecha; 2. Arteria Vesical Caudal; 3. Arteria Uterina Dorsal; 4. Arteria Uterina; 5. Arteria Uterina Lateral Derecha; 6. Arteria Uterina Medial Derecha; 7. Arteria Arco Cervical; 8. Arteria Vesical Media; a. Vejiga; b. Vagina; c. Cérvix. 


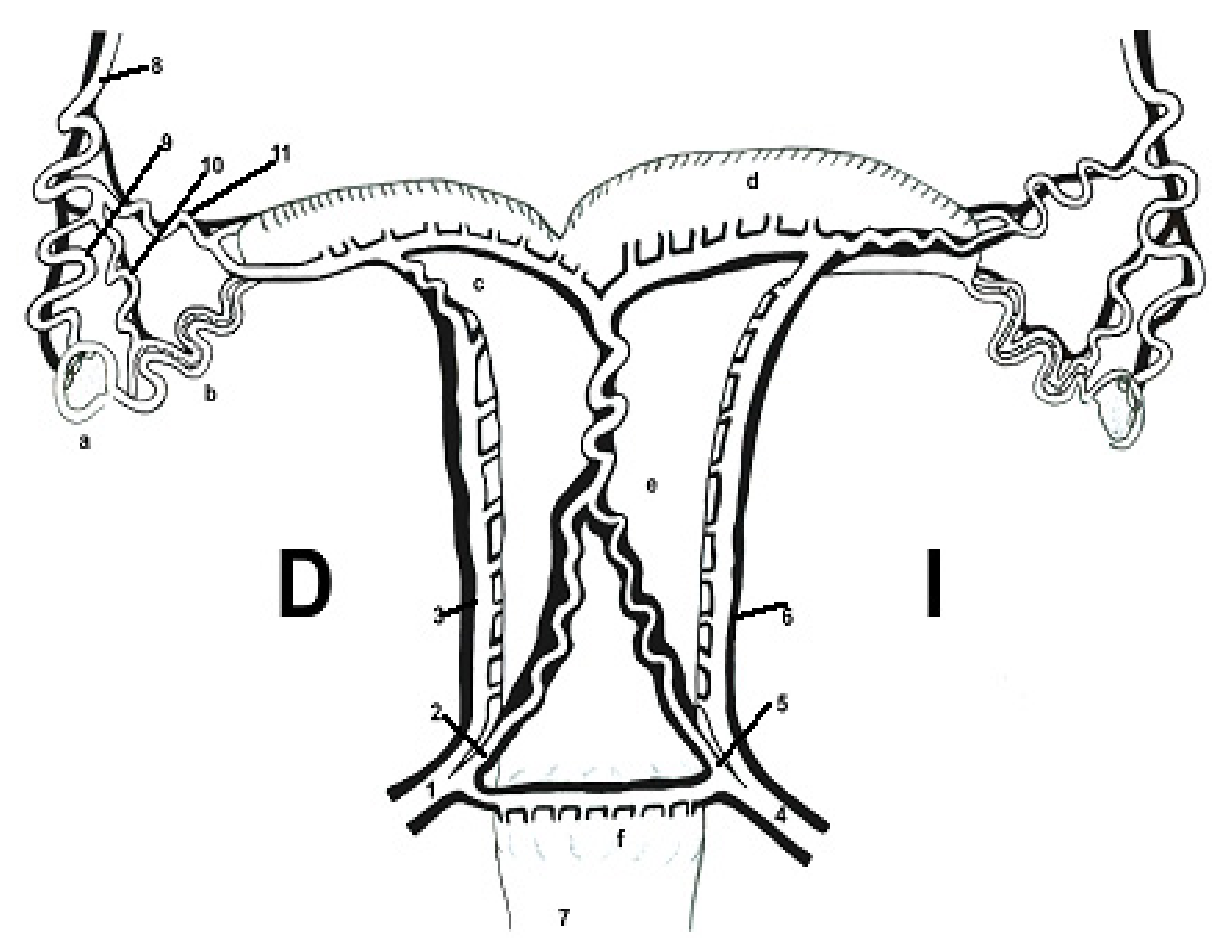

Figura 5. Representación gráfica de la distribución de las arterias y venas del aparato reproductor femenino de la llama, en base a la descripción anatómica realizada. Vista ventral. 1. Arteria Uterina Derecha; 2. Arteria Uterina Medial Derecha; 3. Arteria Uterina Lateral Derecha; 4. Arteria Uterina Izquierda; 5. Arteria Uterina Medial Izquierda; 6. Arteria Uterina Lateral Izquierda; 7. Arteria Arco Cervical; 8. Arteria Ovárica; 9. Rama Ovárica; 10. Rama Tubárica; 11. Rama Uterina; a. Ovario Derecho; b. Oviducto Derecho; c. Cuerno Uterino Derecho; d. Cuerno Uterino Izquierdo; e. Cuerpo Uterino; f. Cérvix

- La distribución de las arterias y venas presentaron un patrón diferente a reportes existentes en otras especies.

- La arteria y vena arco cervical fueron vasos sanguíneos ubicados en la superficie ventral de la cérvix, cuya descripción no existe en especie doméstica o silvestre alguna.

- Se encontraron cinco arterias y venas durante la disección, las cuales no poseen reporte descriptivo alguno (vaginal caudal, vesical media, vaginal craneal, uterina dorsal con sus ramas lateral y medial, y arco cervical).

\section{LiTERATURA Citada}

1. Alzola R, Ghezzi M, Gimeno E. 2004. Topografía y morfología del estómago de llama (Lama glama). Int J Morphol 22(2): 155-164. [Internet], [5 julio 2008]. Disponible en: http://www.scielo.cl/ scielo.php?script=sci_arttext\&pid=S071795022004000200010\&lng=es\&nrm=iso>

2. Ayacucho Portal Regional Agrario. 2008. Ayacucho. CONACS Ayacucho. [Internet], [25 junio 2008]. Disponible en: http://www.agroayacucho.gob.pe/conacs_ayacucho.shtml 
3. Brogliatti G, Palasz A, RodriguezMartínez H, Mapletoft R, Adams G. 2000. Transvaginal collection and ultrastructure of llama (Lama glama) oocytes. Theriogenology 54: 1269-1280.

4. Fowler M. 1998. Medicine and surgery of South American camelids: llama, alpaca, vicuña, guanaco. $2^{\text {nd }}$ ed. Ames: Iowa State University Press. 549 p.

5. Ministerio de Agricultura. 2008. Camélidos Sudamericanos. [Internet], [25 junio 2008]. Disponible en: http:// www.minag.gob.pe/situacion-de-las-actividades-de-crianza-y-produccion/ camelidos-sudamericanos-36.html

6. [ICVGAN] International Committee on Veterinary Gross Anatomical Nomenclature. 2005. Nómina anatómica veterinaria. $5^{\text {th }}$ ed. Knoxville, TN: ICGVAN. 166 p.

7. Sato A, Valencia R, Montoya L. 1986. Revisión anatómica del aparato reproductor de la alpaca hembra (Lama pacos). Rev Camélidos Sudamericanos 2: 4-8.

8. Sato A, Nuñez Q, Valencia R. 1988. Estudio anatómico de las arterias del útero de la alpaca. Rev Camélidos Sudamericanos 6: 3-7.

9. Sato A, Montoya L. 1990. Aparato reproductor de la alpaca (Lama pacos). Rev Camélidos Sudamericanos 7: 5-14.

10. Sisson S, Grossman JD, Getty R. 2000. Anatomía de los animales domésticos. $5^{\text {a }}$ ed. México DF: Ed Masson. 2290 p.

11. Smith CL, Meter AT, Pugh DG. 1994. Reproduction in llamas and alpacas: a review. Theriogenology 41: 573-592.

12. Sumar J. 1981. Rendimiento de canal en llamas. En: IV Convención Internacional sobre Camélidos Sudamericanos. Punta Arenas, Chile.

13. Vilá V, Pérez M, Perozo E, Riera M, Rivera L. 2007. Vascularización del ovario durante el ciclo estral en ovinos. Rev Científica LUZ 17(4): 341-348. 